\title{
The dynamical structure of higher dimensional Chern-Simons theory
}

\author{
Máximo Bañados ${ }^{\mathrm{a}}$, Luis J. Garay ${ }^{\mathrm{b}}$, Marc Henneaux ${ }^{\mathrm{a}, \mathrm{c}, \mathrm{l}}$ \\ a Centro de Estudios Científicos de Santiago, Casilla 16443, Santiago, Chile

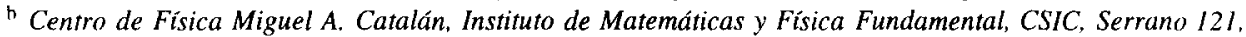 \\ E-28006 Madrid, Spain \\ c Laboratoire de Physique Théorique et Hautes Energies, Universités Paris VI et Paris VII, Bte I26. \\ 4, Place Jussieu, 75252 Paris Cedex 05, France
}

Received 22 May 1996; accepted 17 July 1996

\begin{abstract}
Higher dimensional Chern-Simons theories, even though constructed along the same topological pattern as in $2+1$ dimensions, have been shown recently to have generically a non-vanishing number of degrees of freedom. In this paper, we carry out the complete Dirac Hamiltonian analysis ( separation of first and second class constraints and calculation of the Dirac bracket) for a group $G \times U(1)$. We also study the algebra of surface charges that arise in the presence of boundaries and show that it is isomorphic to the $\mathrm{WZW}_{4}$ discussed in the literature. Some applications are then considered. It is shown, in particular, that Chern-Simons gravity in dimensions greater than or equal to five has a propagating torsion.
\end{abstract}

PACS: 04.20.Fy; 11.10.Kk

Keywords: Chern-Simons theory; Dirac brackets; Lovelock gravity; Surface charges

\section{Introduction}

In a previous paper [1], we have shown that pure Chern-Simons theories in spacetime dimensions greater than or equal to five possess local degrees of freedom in contrast to the familiar three-dimensional case. The only exception is the Chern-Simons theory based on the one-dimensional group $U(1)$, which is devoid of local degrees of freedom for any space-time dimension. However, whenever the gauge group is of dimension

\footnotetext{
${ }^{1}$ Permanent address: Université Libre de Bruxelles, Campus Plaine, C.P. 231, B-1050, Bruxelles, Belgium. 
greater than 1 , the Chern-Simons action generically contains propagating degrees of freedom.

One way to understand this somewhat unexpected result is to observe that the equations of motion no longer imply that the curvature vanishes for dimensions greater than or equal to five. If there is only one gauge field, i.e., if the gauge group is $G=U(1)$, one can always bring its curvature $F$ to some fixed canonical form by using the diffeomorphism invariance (Darboux theorem). Thus, even though $F \neq 0$, one may assume that it has a fixed form and, therefore, the space of solutions of the equations of motion modulo gauge transformations is reduced to a single point. If, however, the gauge group is larger, there are more curvatures. One may bring one of them to a fixed canonical form as for $U(1)$, but once this is done, there is not enough invariance left to fix the other curvatures in a similar way. Thus, the space of solutions is now bigger and, consequently, there exist local degrees of freedom.

The number of local degrees of freedom was explicitly counted in Ref. [1] by using the Hamiltonian formalism. The phase space of the theory was constructed and all the constraints were exhibited. We also derived the number of second and first class constraints. This provided the necessary information to count the number of local degrees of freedom according to the formula

$$
\mathcal{N}=\frac{1}{2}(P-2 F-S),
$$

where $P$ is the dimension of phase space, $F$ is the number of first class constraints and $S$ is the number of second class constraints (see, e.g., Ref. [2]).

Although the analysis of Ref. [1] enables one to conclude rigorously to the existence of local degrees of freedom, the second class constraints were not explicitly separated from the first class ones; only their number was given and the Dirac bracket associated to the elimination of the second class constraints was not computed either. As it is known, these steps are quite important and must be carried out before trying to quantize theory.

An interesting feature of higher dimensional Chern-Simons theories, also displayed in Ref. [1], is that these are theories invariant under space-time diffeomorphisms for which the generator $\mathcal{H}(\boldsymbol{x})$ of time-like diffeomorphisms is not independent from the other constraints. That is, the time-like diffeomorphisms can be expressed in terms of the space-like diffeomorphisms and of the internal gauge transformations. Now, it is well known that the 'super-Hamiltonian' constraint $\mathcal{H}(\boldsymbol{x})|\psi\rangle=0$ is usually the one that resists an exact treatment in the quantum theory. This appears quite strikingly in the loop representation approach to quantum gravity [3]. In the case of higher dimensional Chern-Simons theories, however, the 'hard constraints' are absent, even though there are local degrees of freedom. Thus, any state that is invariant under both the internal gauge symmetries and the space-like diffeomorphisms is automatically a solution of all the quantum constraint equations. Solving the 'kinematical' constraints associated with space-like diffeomorphism invariance and internal gauge invariance, is, however, not entirely straightforward in higher dimensional Chern-Simons theories. This is because the components of the connection have non-trivial Dirac brackets and thus do not define 
simultaneously diagonalizable operators. It makes the issue of computing these brackets even more pressing.

In this paper, we complete the canonical analysis of higher dimensional Chern-Simons theory. It turns out that the separation of first and second class constraints is technically intricate for an arbitrary gauge group $G$. However, if one considers a Chern-Simons action for the gauge group $G \times U(1)$, the calculations become much simpler. The only requirement on the group $G$ is that it possesses a non-degenerate bilinear invariant form. In order to avoid uninteresting (and conceptually trivial) complications, we shall therefore complete the Dirac bracket analysis only in that case, as well as in the $U(1)$ case, which has its own peculiarities.

Three-dimensional Chern-Simons theory is well known to induce a rich dynamics at the boundary [4]. One may therefore wonder whether this is also the case in higher dimensional space-times. The answer to this question is affirmative. This problem has already been considered in the literature [5,6]. Specifically, in Ref. [6], the connection between a 'conformal' field theory in four dimensions and a Kähler five-dimensional Chern-Simons theory was established. In this work, we explicitly exhibit the symmetry algebra arising at the boundary for the full Chern-Simons theory with no extra assumption other than the boundary conditions. For definiteness, we consider Chern-Simons theory in five dimensions and show that, if the gauge group is taken to be $G \times U(1)$, as above, then the resulting symmetry algebra is just the $\mathrm{WZW}_{4}$ algebra (based on $G$ ) discussed in Ref. [6], with the curvature of the $U(1)$ factor appearing as a Kähler form. (For a recent work dealing with the $\mathrm{WZW}_{4}$ algebra see Ref. [7].)

This paper is organized as follows. After a brief survey of our conventions, we review in Section 2 the results of Ref. [1]. We recall, in particular, the importance of the so-called generic condition that was introduced there. Then, we illustrate the generic condition in the physically interesting context of Lovelock-Chern-Simons gravity (Section 3), as well as for some seven-dimensional Chern-Simons theories (Section 4). In Section 5, we complete the Dirac analysis for the $U(1)$ theory, which has no degrees of freedom. We show that in this theory one needs to break general covariance in order to separate the first and second class constraints. This is quite analogous to what happens for the superparticle [8-10] and the analogies are pointed out. We turn next (Section 6) to the separation of first and second class constraints in the more general theory with a gauge group $G \times U(1)$ and we work out the Dirac bracket between the basic dynamical variables. In contrast to the $U(1)$-case, the analysis can be performed without breaking manifest covariance, by taking advantage of the peculiar group structure. Finally we discuss the global charges arising when the spatial manifold has a boundary, and show that they fulfill, in five dimensions, the $\mathrm{WZW}_{4}$ algebra found in Ref. [6]. We summarize and conclude in Section 8 . 


\section{Local dynamics}

\subsection{Conventions and definitions}

The Chern-Simons action in higher odd dimensions is a direct generalization of the three-dimensional case. Let us consider a Lie algebra $G$ of dimension $N$. Let $\tilde{F}^{a}$ be the curvature 2-form ${ }^{2} \tilde{F}^{a}=d A^{a}+\frac{1}{2} f_{b c}^{a} A^{b} \wedge A^{c}$ associated to the gauge field 1-form $A^{a}$, where $f_{b c}^{a}$ are the structure constants of the gauge group, and let $g_{a_{1} \ldots a_{n+1}}$ be a rank $n+1$, symmetric tensor invariant under the adjoint action of the gauge group. The Chern-Simons Lagrangian in $D=2 n+1$ dimensions $\mathcal{L}^{2 n+1}$ is defined through the formula

$$
d \mathcal{L}^{2 n+1}=g_{a_{1} \ldots a_{n+1}} \tilde{F}^{a_{1}} \wedge \cdots \wedge \tilde{F}^{a_{n+1}}
$$

The Chern-Simons action $I=\int_{M} \mathcal{L}_{\mathrm{CS}}^{2 n+1}$ is invariant, up to a boundary term, under standard gauge transformations

$$
\delta_{\epsilon} A_{\mu}^{a}=-D_{\mu} \epsilon^{a}
$$

It is also invariant under diffeomorphisms on the space-time manifold $M, \delta_{\eta} A_{\mu}^{a}=£_{\eta} A_{\mu}^{a}$, because $\mathcal{L}_{\mathrm{CS}}^{2 n+1}$ is a $(2 n+1)$-form. The diffeomorphisms on $M$ can be represented equivalently by

$$
\delta_{\eta} A_{\mu}^{a}=-\eta^{\nu} F_{\mu \nu}^{a}
$$

This transformation differs from the Lie derivative only by a gauge transformation and it is often called improved diffeomorphism [11].

If the only symmetries of the Chern-Simons action are the diffeomorphisms (2.3) and the gauge transformations (2.2), then we shall say that there is no accidental gauge symmetry. How this translates into an algebraic condition on the invariant tensor $g_{a_{1} \ldots a_{n+1}}$ will be described precisely in Section 2.4. As we shall also indicate, the absence of accidental gauge symmetries is 'generic'. Generic, however, does not mean universal and there exist examples with further gauge symmetries. A typical one is obtained by taking all the mixed components of $g_{a_{1} \ldots a_{n+1}}$ equal to zero, so that the action is just the direct sum of $N$ copies of the action for a single Abelian field. This theory is then clearly invariant under diffeomorphisms acting independently on each copy. But there is no reason to take vanishing mixed components for $g_{a_{1} \ldots a_{n+1}}$. In fact, for a non-Abelian theory, the form of the invariant tensor is severely restricted. If the mixed components of $g_{a_{1} \ldots a_{n+1}}$ differ from zero (and cannot be brought to zero by a change of basis), then the action is not invariant under diffeomorphisms acting independently on each gauge field component $A^{a}$, because the invariance of the cross terms requires the diffeomorphism

\footnotetext{
${ }^{2}$ We denote by $\tilde{F}^{a}=\frac{1}{2} F_{\mu \nu}^{a} d x^{\mu} \wedge d x^{\nu}$ the space-time curvature 2 -form (greek indices run over space-time while latin indices run over the space-like hypersurfaces). The symbol $F^{\prime l}$ will denote the space-like curvature 2-form, $F^{a}=\frac{1}{2} F_{i j}^{a} d x^{i} \wedge d x^{j}$.
} 
parameters for each copy to be equal, thus gluing all of them together in a single symmetry.

The Chern-Simons equations of motion are easily found to be

$$
g_{c a a_{1} \ldots a_{n}} \tilde{F}^{a_{1}} \wedge \cdots \wedge \tilde{F}^{a_{n}}=0
$$

and they reduce to $\tilde{F}^{a}=0$ only in the three-dimensional case (provided of course that $g_{a b}$ is invertible).

\subsection{The Hamiltonian action}

In order to perform the Hamiltonian analysis, we assume that the space-time manifold $M$ has the topology $\mathbb{R} \times \Sigma$, where $\Sigma$ is a $2 n$-dimensional manifold. In this section, we will concentrate on the local properties of the theory, that is, we will not analyze the special features that arise if $\Sigma$ has a boundary or a non-trivial topology. The presence of boundaries will be considered in Section 7. We decompose the space-time gauge field 1 -form $A^{a}$ as $A_{\mu}^{a} d x^{\mu}=A_{0}^{a} d t+A_{i}^{a} d x^{i}$ where the coordinate $t$ runs over $\mathbb{R}$ and the $x^{i}$ are coordinates on $\Sigma$. Although there is no space-time metric to give any meaning to expressions such as time-like or space-like, we will call time the coordinate $t$ and we will say that $\Sigma$ is a space-like section as shorthand expressions.

It is easy to see that the Chern-Simons action depends linearly on the time derivative of $A_{i}^{a}$,

$$
I=\int_{\mathbb{R}} \int_{\Sigma}\left[l_{a}^{i}\left(A_{j}^{b}\right) \dot{A}_{i}^{a}-A_{0}^{a} K_{a}\right],
$$

where $K_{a}$ is given by

$$
K_{a}=-\frac{1}{2^{n} n} g_{a a_{1} a_{2} \ldots a_{n}} \epsilon^{i_{1} \ldots i_{2 n}} F_{i_{1} i_{2}}^{a_{1}} \cdots F_{i_{2 n-1} i_{2 n}}^{a_{n}} .
$$

The explicit form of the function $l_{a}^{i}\left(A_{j}^{b}\right)$ appearing in Eq. (2.5) is not needed here but only its 'exterior' derivative in the space of spatial connections, which reads

$$
\begin{aligned}
\Omega_{a b}^{i j} & \equiv \frac{\delta l_{b}^{j}}{\delta A_{i}^{a}}-\frac{\delta l_{a}^{i}}{\delta A_{j}^{b}} \\
& =-\frac{1}{2^{n-1}} \epsilon^{i j i_{1} \ldots i_{2 n-2}} g_{a b a_{1} \ldots a_{n-1}} F_{i_{1} i_{2}}^{a_{1}} \ldots F_{i_{2 n-3} i_{2 n-2}}^{a_{n-1}}
\end{aligned}
$$

The equations of motion obtained by varying the action (2.5) with respect to $A_{i}^{d t}$ are given by

$$
\Omega_{a b}^{i j} \dot{A}_{j}^{b}=\Omega_{a b}^{i j} D_{j} A_{0}^{b},
$$

while the variation of the action with respect to $A_{0}^{a}$ yields the constraint

$$
K_{a}=0
$$


Of course, Eqs. (2.8) and (2.9) are completely equivalent to Eq. (2.4). Despite the fact that Eqs. (2.8) are first order, they are not Hamiltonian. The reason is that the matrix $\Omega_{a b}^{i j}$ is not invertible, as we will see. Indeed, on the surface defined by the constraint (2.9), the matrix $\Omega$ has, at least, $2 n$ null eigenvectors. The non-invertibility of $\Omega$ is a signal of a gauge symmetry. We shall see below that this symmetry is nothing but the diffeomorphism invariance.

To proceed with the Hamiltonian formulation of the action we shall use the Dirac method [2]. Since the action (2.5) is linear in the time derivatives of $A_{i}^{a}$, the canonically conjugate momenta $p_{a}^{i}$ are subject to the $2 n N$ primary constraints,

$$
\phi_{a}^{i}=p_{a}^{i}-l_{a}^{i} \approx 0
$$

These constraints transform in the coadjoint representation of the Lie algebra because the inhomogeneous terms in the transformation laws of $p_{a}^{i}$ and $l_{a}^{i}$ cancel out.

In principle one should also define a canonical momentum for $A_{0}^{a}$. This would generate another constraint, $p_{a}^{0}=0$, whose consistency condition yields the constraint $K_{a}=0$. The constraint $p_{a}^{0}=0$ is first class and generates arbitrary displacements of $A_{0}^{a}$. One can drop $p_{0}^{a}$ and keep $A_{0}^{a}$ as an arbitrary Lagrange multiplier for the constraint $K_{a}=0$.

It turns out to be more convenient to replace the constraints $K_{a}$ by the equivalent set

$$
G_{a}=-K_{a}+D_{i} \phi_{a}^{i}
$$

This redefinition is permissible because the surface defined by $K_{a}=0, \phi_{i}^{a}=0$ is equivalent to the surface defined by $G_{a}=0, \phi_{i}^{a}=0$. The motivation to replace $K_{a}$ by $G_{a}$ is that the new constraints $G_{a}$ generate the gauge transformations (2.2) and therefore are first class. Indeed one can easily check that

$$
\delta A_{i}^{a}=\left\{A_{i}^{a}, \int_{\Sigma} \lambda^{b} G_{b}\right\}=-D_{i} \lambda^{a} .
$$

The Hamiltonian action takes the form

$$
I=\int_{\mathbb{R}} \int_{\Sigma}\left[p_{a}^{i} \dot{A}_{i}^{a}-A_{0}^{a} G_{a}-u_{i}^{a} \phi_{a}^{i}\right],
$$

where the Poisson brackets among the constraints are given by

$$
\begin{aligned}
& \left\{\phi_{a}^{i}, \phi_{b}^{j}\right\}=\Omega_{a b}^{i j}, \\
& \left\{\phi_{a}^{i}, G_{b}\right\}=f_{a b}^{c} \phi_{c}^{i}, \\
& \left\{G_{a}, G_{b}\right\}=f_{a b}^{c} G_{c} .
\end{aligned}
$$

Here, $f_{a b}^{c}$ are the structure constants of the Lie algebra under consideration.

It follows from the constraint algebra that there are no further constraints. The consistency condition

$$
\dot{G}_{a}=0
$$


is automatically fulfilled because $G_{a}$ is first class while the other consistency equation

$$
\dot{\phi}_{a}^{i}=\Omega_{a b}^{i j} u_{j}^{b}=0
$$

will just restrict some of the Lagrange multipliers $u_{j}^{b}$.

\subsection{First class constraints}

Eqs. (2.15) and (2.16) reflect that the constraints $G_{a}$ are the generators of the gauge transformations and that the constraints $\phi_{a}^{i}$ transform in the coadjoint representation. This means, in particular, that the $G_{a}$ 's are first class, as mentioned above.

The nature of the constraints $\phi_{a}^{i}$ is determined by the eigenvalues of the matrix $\Omega_{a b}^{i j}$. It turns out that the matrix $\Omega_{a b}^{i j}$ is not invertible on the constraint surface and, therefore, not all the $\phi$ 's are second class. Indeed, using some simple combinatorial identities, one can prove that $K_{a}$ and $\Omega_{a b}^{i j}$ satisfy the identity

$$
\Omega_{a b}^{i j} F_{k j}^{b}=\delta_{k}^{i} K_{a}
$$

This equation shows that, on the constraint surface $K_{a}=0$, the matrix $\Omega_{a b}^{i j}$ has, at least, $2 n$ null eigenvectors $\left(v_{k}\right)_{j}^{b}=F_{k j}^{b},(k=1, \ldots, 2 n)$. The existence of these $2 n$ null eigenvectors of $\Omega$ tells us that among the $\phi$ 's, there are $2 n$ first class constraints. These constraints are given by

$$
H_{i} \equiv F_{i j}^{a} \phi_{a}^{j}
$$

and they generate the spatial diffeomorphisms (2.3), namely, $\delta A_{i}^{a}=\left\{A_{i}^{a}, \int_{2} \eta^{j} H_{j}\right\}=$ $-\eta^{j} F_{i j}$. Thus, they satisfy the spatial diffeomorphism algebra, up to gauge transformations. The presence of these constraints is not surprising because the Chern-Simons action is invariant under diffeomorphisms for any choice of the invariant tensor $g_{a_{1} \ldots a_{n+1}}$. What is perhaps more surprising in view of what occurs for ordinary Chern-Simons theory in three dimensions, is that the constraints (2.20) are generically independent from the constraints $G_{a}=0$ generating local internal gauge transformations ( $F_{i j}^{a} \neq 0$, see below).

One could also expect the presence of another first class constraint, namely, the generator of time-like diffeomorphisms. However, this symmetry is not independent from the other ones and hence its generator is a combination of the first class constraints $G_{a}$ and $H_{i}$. This can be viewed as follows. The action of a time-like diffeomorphism parameterized by $\xi^{\mu}=\left(\xi^{0}, 0\right)$ acting on $A_{i}^{a}$ is (see Eq. (2.3))

$$
\delta_{\xi} A_{i}^{a}=-\xi^{0} F_{i 0}^{a}
$$

Now, the equations of motion (2.8) are $\Omega_{a b}^{i j} F_{0 j}^{b}=0$. Let us assume (this assumption will be clarified below) that the only null eigenvectors of $\Omega$ are those given above, then there must exist some $\zeta^{k}$ such that $F_{j 0}^{b}=\zeta^{k} F_{j k}^{b}$. Inserting this result in Eq. (2.21), we obtain

$$
\delta_{\xi} A_{i}^{a}=-\xi^{0} \zeta^{k} F_{i k}^{a}
$$


which is a spatial diffeomorphism with parameter $\xi^{0} \zeta^{k}$. Hence, time-like diffeomorphisms are equivalent to space-like diffeomorphisms on-shell. For that reason, there is no constraint associated to normal deformations of the surface. If the theory has 'accidental symmetries', i.e. the matrix $\Omega$ has more zero eigenvalues, the above analysis shows that the time-like diffeomorphisms can still be written in terms of spatial diffeomorphisms plus the extra 'accidental' symmetries.

\subsection{Generic theories}

We now examine whether the first class constraints $G_{a}$ and $H_{i}$ are independent and constitute a complete set. At this point, we must distinguish between the $N=1$ and $N>1$ cases. Indeed, it turns out that for the Lie algebra $u(1)$, the theory cannot be obtained as a limiting case from the $N>1$ theory.

Consider first the general case with $N>1$. The eigenvalues of $\Omega$, which determine the nature of the constraints $\phi_{a}^{i}$, depend on the properties of the invariant tensor $g_{a_{1} \ldots a_{n+1}}$ and, for a definite choice of $g_{a_{1} \ldots a_{n+1}}$, they also depend on the phase space location of the system since the constraint surface of the Chern-Simons theory is stratified into phase space regions where the matrix $\Omega_{a b}^{i j}$ has different ranks. For example, $F^{a}=0$ is always a solution of the equations of motion and, for that solution, $\Omega$ is identically zero. There exist, however, other solutions of the equations of motion for which $\Omega \neq 0$. The rank of $\Omega$ classifies the phase space into regions with different numbers of local degrees of freedom. The key ingredient controlling the maximum possible rank of $\Omega$ is the algebraic structure of the invariant tensor $g_{a_{1} \ldots a_{n+1}}$.

We will say that an invariant tensor $g_{a_{1} \ldots a_{n+1}}$ is generic if and only if it satisfies the following condition: There exist solutions $F_{i j}^{a}$ of the constraints $K_{a}=0$ such that

(i) the matrix $F_{k j}^{b}$ (with $b, j$ as row index and $k$ as column index) has maximum rank $2 n$, so that the only solution of $\xi^{k} F_{k j}^{b}=0$ is $\xi^{k}=0$ and therefore the $2 n$ null eigenvectors $\left(v_{k}\right)_{j}^{b}=F_{k j}^{b}(k=1, \ldots, 2 n)$ are linearly independent;

(ii) the $(2 n N) \times(2 n N)$ matrix $\Omega_{a b}^{i j}$ has the maximum rank compatible with (i), namely $2 n N-2 n$; in other words, it has no other null eigenvectors besides $\left(v_{k}\right)_{j}^{b}=F_{k j}^{b}$ $(k=1, \ldots, 2 n)$.

We will also say that the solutions $F_{i j}^{a}$ of the constraints $K_{a}=0$ such that (i) and (ii) hold are generic. The reason for this name comes from the following observation. For a given generic tensor $g_{a_{1} \ldots a_{n+1}}$, a solution fulfilling both conditions (i) and (ii) will still fulfill them upon small perturbations, since maximum rank conditions correspond to inequalities and therefore, they define open regions. Conversely, a solution not fulfilling conditions (i) or (ii), i.e. located on the surface where lower ranks are achieved (defined by equations expressing that some non-trivial determinants vanish), will fail to remain on that surface upon generic perturbations consistent with the constraints. Thus, non-generic solutions belong to subsets of the constraint surface of smaller dimension.

The physical meaning of the above algebraic conditions is straightforward. They simply express that the gauge transformations (2.2) and the spatial diffeomorphisms 
(2.3) are independent and that the $H_{i}$ are the only first class constraints among the $\phi_{a}^{j}$ 's.

As we stressed above, the $N=1$ theory cannot be obtained as a limiting case from the $N>1$ theory. The definition of what is meant by 'generic' in the $N=1$ case must therefore be amended as follows. For $N=1$, the invariant tensor has a single component $g_{1 \ldots 1}$, which we assume, of course, to be different from zero. The constraint is then

$$
\epsilon^{i_{1} \ldots i_{2 n}} F_{i_{1} i_{2}} \cdots F_{i_{2 n-1} i_{2 n}}=0
$$

which implies that the matrix $F_{i j}$ cannot be invertible. The solutions of Eq. (2.23) are the set of matrices $F_{i j}$ with zero determinant. Thus, the equation $\xi^{i} F_{i j}=0$ does not imply $\xi^{i}=0$ and the constraint (2.23) prevents us from finding solutions of the $N=1$ theory satisfying the generic condition of the $N>1$ case. This means, in particular, that the $2 n$ spatial diffeomorphisms are not all independent. Solutions of Eq. (2.23) such that $F_{i j}$ has the maximum rank compatible with the constraint $(2.23)$ will be called generic. This rank is clearly $2 n-2$. The complete Hamiltonian analysis for the $N=1$ theory is performed in Section 5.

\subsection{Degrees of freedom count $(N>1)$}

When the generic condition is satisfied, the count of local degrees of freedom goes as follows. We have $2 \times 2 n N$ canonical variables $\left(A_{i}^{a}, p_{a}^{i}\right), N$ first class constraints $G_{a}$ associated with the gauge invariance, $2 n$ first class constraints $H_{i}$ associated with the spatial diffeomorphism invariance, and $2 n N-2 n$ second class constraints (the remaining $\phi_{a}^{i}$ ). Hence, we have

$$
\begin{aligned}
\mathcal{N} & =\frac{1}{2}[4 n N-2(N+2 n)-(2 n N-2 n)] \\
& =n N-n-N
\end{aligned}
$$

local degrees of freedom (for $n>1, N>1$ ). It should be stressed here that this formula gives the number of local degrees of freedom associated to the open region of phase space defined by generic solutions.

This formula does not apply to $N=1$ because the spatial diffeomorphisms are not independent in that case. One finds instead that there are no local degrees of freedom ( see Section 5). For a similar reason, this formula does not apply to $n=1 \quad(D=3)$ where diffeomorphism invariance is completely contained within the ordinary YangMills gauge invariance.

\subsection{The generic condition in the Lagrangian equations of motion}

It is instructive to study the implications of the generic condition in the context of the Lagrangian equations of motion. This provides also an equivalent method of counting the number of local degrees of freedom. The Lagrangian equations of motion written in 
a covariant way are given in Eq. (2.4). Upon a $(2 n+1)$-decomposition, these equations acquire the expressions given in Eqs. (2.8) and (2.9).

If the generic condition is fulfilled, then Eq. (2.8) can be rewritten in the useful and simple form,

$$
\dot{A}_{i}^{a}=D_{i} A_{0}^{a}+N^{k} F_{k i}^{a},
$$

where the $N^{k}$ are $2 n$ arbitrary functions of space-time. This form of the equations of motion clearly shows that the time evolution is generated by a gauge transformation (with parameter $A_{0}^{a}$ ) plus a diffeomorphism (with parameter $N^{l}$ ). (Eq. (2.25) follows directly from Eq. (2.8), and the fact that, in the generic case, $\Omega$ has only $2 n$ null eigenvectors given in Eq. (2.19).) Therefore, the Lagrangian equations of motion can be replaced by the constraint (2.9) plus Eq. (2.25). Due to the simplicity of Eq. (2.25) we can study its space of solutions, modulo gauge transformations.

Eq. (2.25) is invariant under standard gauge transformations,

$$
\delta_{\lambda} A_{i}^{a}=-D_{i} \lambda^{a}, \quad \delta_{\lambda} A_{0}^{a}=-\dot{\lambda}^{a}-\left[\lambda, A_{0}\right]^{a}, \quad \delta_{\lambda} N^{k}=0,
$$

where $[\cdot, \cdot]$ is the commutator in the Lie algebra. Eq. (2.25) is also invariant under spatial diffeomorphisms

$$
\delta_{\xi} A_{i}^{a}=-\xi^{j} F_{i j}^{a}, \quad \delta_{\xi} A_{0}^{a}=-\xi^{j} F_{0 j}^{a}, \quad \delta_{\xi} N^{i}=\dot{\xi}^{i}+[\xi, N]^{i},
$$

where now the symbol $[\cdot, \cdot]$ denotes the Lie bracket of two spatial vectors. Of course, the constraint Eq. (2.9) is also invariant under both gauge transformations and diffeomorphisms.

We shall study the solutions of the equations of motion in the gauge

$$
A_{0}^{a}=0, \quad N^{k}=0 \quad \text { (time gauge) } \text {. }
$$

In this gauge, Eq. (2.25) simply says that $\dot{A}_{i}^{a}=0$, hence, the configurations are time independent. It is important to note that the above gauge choice does not exhaust all the gauge symmetry. The conditions (2.28) are preserved by gauge transformations and diffeomorphism that do not depend on time, i.e., by transformations whose parameters satisfy $\dot{\lambda}^{a}=0$ and $\dot{\xi}^{i}=0$.

Thus, in the time gauge, we are left only with the constraint Eq. (2.9) with the extra condition that the fields are time independent. Eq. (2.9) is invariant under the residual gauge group consisting in time-independent gauge transformations and diffeomorphisms. In summary, we have $2 n N$ arbitrary functions of the spatial coordinates, $A_{i}^{a}\left(x^{i}\right)$. These functions are restricted by $N$ equations, the constraints (2.9). Also, there is a $(N+2 n)$ dimensional residual gauge group which can be used to set $N+2 n$ functions equal to zero.

Therefore, the number of arbitrary functions in the solutions of the equations of motion is

$$
2 n N-N-(N+2 n)=2(n N-N-n) .
$$


These are the Lagrangian 'integration functions' for the equations of motion, which are twice the number of local degrees of freedom, in agreement with Eq. (2.24).

\section{Lovelock-Chern-Simons gravity}

The goal of this section is twofold. On the one hand, we exhibit the Lovelock-Chern-Simons theory as a concrete example with a non-Abelian internal group for which the generic condition defined in Section 2.4 is fulfilled. On the other hand, the analysis of the dynamics of the Lovelock-Chern-Simons action reveals a rather unexpected result. It turns out that the torsion tensor is dynamical in this theory, hence the Palatini and second order formalisms are not equivalent in higher dimensional Chern-Simons gravity, contrary to what happens in three dimensions.

The Lovelock-Chern-Simons theory (in higher dimensions) is not defined by the Hilbert-Einstein action, but, rather, it contains higher powers of the curvature tensor. However, the equations of motion are first order in the tetrad and spin connection, and, if the torsion is set equal to zero, they are second order in the metric. The gravitational Chern-Simons action is a particular case of the so-called Lovelock action [12]. For this reason, we call this theory the Lovelock-Chern-Simons (LCS) theory. It is a natural extension to higher dimensions of the formulation of $2+1$ gravity given by Achúcarro and Townsend [13] and Witten [14]. As we shall see, however, the dynamical content is quite different. The construction of the Lovelock-Chern-Simons theory has been carried out in Ref. [15]. Here we briefly review its main features.

Let $A^{A B}$ be a connection for the group $S O(2 n, 2)$ (we recall that $D=2 n+1$ ) and $F^{A B}$ its curvature 2 -form (here, the capital indices $A, B, \ldots$ run over $1,2, \ldots, 2 n+2$ ). The connection $A^{A B}$ can be split in the form

$$
A^{A B}=\left(\begin{array}{cc}
w^{a b} & e^{a} / l \\
-e^{b} / l & 0
\end{array}\right),
$$

where $l$ parameterizes the cosmological constant and $w^{a b}$ and $e^{a}$ transform, respectively, as a connection and as a vector under the action of the Lorentz subgroup $\operatorname{SO}(2 n, 1)$. Hence, $w^{a b}$ will be called the spin connection and $e^{a}$ the vielbein. Similarly, the curvature has the form

$$
F^{A B}=\left(\begin{array}{cc}
F^{a b} & T^{a} / l \\
-T^{b} / l & 0
\end{array}\right),
$$

where $F^{a b}=R^{a b}+\left(1 / l^{2}\right) e^{a} \wedge e^{b}$ and $T^{a}=D e^{a}$ is the torsion tensor.

The $S O(2 n, 2)$ Chern-Simons Lagrangian is defined by making use of the Levi-Civita invariant tensor,

$$
d \mathcal{L}_{\mathrm{LCS}}=\epsilon_{A_{1} \ldots A_{2 n+2}} F^{A_{1} A_{2}} \wedge \cdots \wedge F^{A_{2 n+1} A_{2 n+2}} .
$$

Since $\epsilon_{A_{1} \ldots A_{2 n 12}}$ is an invariant tensor of $S O(2 n, 2)$, we say that $\mathcal{L}_{\mathrm{LCS}}$ is a Chern-Simons Lagrangian for the (adS) group $S O(2 n, 2)$. When written in terms of the vielbein and 
spin connection, the Lagrangian defined in Eq. (3.3) is a particular case of the Lovelock Lagrangian considered in Ref. [12]. Black hole solutions for this action have been found in Ref. [16].

The equations of motion for this theory are

$$
\epsilon_{A B A_{1} \ldots A_{2 n}} F^{A_{1} A_{2}} \wedge \cdots \wedge F^{A_{2 n-1} A_{2 n}}=0,
$$

which are explicitly invariant under $S O(2 n, 2)$. Splitting the curvature $F^{A B}$ as in Eq. (3.2), these equations separate into the two sets of equations

$$
\begin{aligned}
\epsilon_{a a_{1} \ldots a_{2 n}} F^{a_{1} a_{2}} \wedge \cdots \wedge F^{a_{2 n-1} a_{2 n}} & =0, \\
\epsilon_{a b a_{1} \ldots a_{2 n-1}} F^{a_{1} a_{2}} \wedge \cdots \wedge F^{a_{2 n-3} a_{2 n-2} \wedge} T^{a_{2 n-1}} & =0,
\end{aligned}
$$

which are the equations of motion following from varying the action with respect to the vielbein and spin connection, respectively. After the split has been made, the equations are explicitly invariant only under $S O(2 n, 1)$, but, in view of Eq. (3.4), they are in fact invariant under the larger group $S O(2 n, 2)$. Note that, as mentioned above, $T^{a}=0$ is always a solution of Eq. (3.6) and, in $2+1$ dimensions, it is the only solution. We now prove that, for $n>1$, this is not the most general solution and dynamical $T^{a} \neq 0$ modes exist.

For simplicity, we consider the five-dimensional case. As it has been shown in Ref. [17], the Lovelock Lagrangian written in the second order formalism $\left(T^{a}=0\right)$ has the same number of degrees of freedom as the Hilbert Lagrangian. Thus, in five dimensions, the theory with zero torsion carries $D(D-3) / 2=5$ local (physical) degrees of freedom. On the other hand, since the Lagrangian $\mathcal{L}_{\mathrm{LCS}}$ defines a Chern-Simons theory we can count the number of degrees of freedom by using the formula (2.24). However, before we can apply that formula we need to prove that the Lovelock-Chern-Simons theory is generic in the sense defined in Section 2.4.

The constraint for this theory is

$$
\epsilon_{A B C D E F} F^{C D} \wedge F^{E F}=0 \text {, }
$$

where $F^{C D}=\frac{1}{2} F_{i j}^{C D} d x^{i} \wedge d x^{j}$ are the spatial projections of the 2-form curvature. To prove that this theory is generic, it is enough to find one solution for which the matrix $\Omega$ has maximum rank. The 2 -form curvature given by

$$
\begin{aligned}
& F^{12}=d x^{1} \wedge d x^{2}+d x^{3} \wedge d x^{4}, \\
& F^{34}=d x^{1} \wedge d x^{2}-d x^{3} \wedge d x^{4}, \\
& F^{56}=d x^{1} \wedge d x^{3}+d x^{2} \wedge d x^{4},
\end{aligned}
$$

with all other components equal to zero satisfies the constraint (3.7) and has maximum rank. The proof of this statement is straightforward. One looks at the equation

$$
\epsilon_{A B C D E F} F^{C D} \wedge V^{E F}=0
$$


where $V^{E F}$ is a one-form. Due to the fact that $F^{12}, F^{34}$ and $F^{56}$ are non-degenerate 2 -forms, one easily obtains that this equation possesses only four independent solutions, that is, $\Omega$ has the maximum rank.

Note that $F^{A B}$ given in $\mathrm{Eq}$. (3.8) does not have any zero column in the indices $(A, B)$ therefore this solution clearly has a non-zero torsion (see Eq. (3.2)). Note also that the above curvature can be derived from the connection $W^{A B}$,

$$
\begin{aligned}
& W^{12}=x^{1} d x^{2}+x^{3} d x^{4}, \\
& W^{34}=x^{1} d x^{2}-x^{3} d x^{4}, \\
& W^{56}=x^{1} d x^{3}+x^{2} d x^{4},
\end{aligned}
$$

with all other components equal to zero. Thus, $F^{A B}$ given in Eq. (3.8) represents an allowed physical configuration.

The existence of the above solution ensures that this theory is generic and therefore we can apply the formula (2.24) to count the number of degrees of freedom. In five dimensions, the Lovelock-Chern-Simons theory is a Chern-Simons theory for the Lie algebra $S O(4,2)$, of dimension 15 . Hence, formula (2.24) gives $2 \times 15-15-2=13$ local degrees of freedom. Thus, this theory indeed has more degrees of freedom than the metric theory. This reflects the fact that setting the torsion equal to zero eliminates degrees of freedom. In other words, Lovelock theory, at least in the case considered here, has a dynamical torsion.

\section{The seven-dimensional case}

In Ref. [1], examples fulfilling the generic condition were explicitly given only in five dimensions. We exhibit in this section seven-dimensional examples for which the generic condition is satisfied. In this case, there exists a 'simple' choice for the invariant tensor: We can take the rank-four invariant symmetric tensor $g_{a b c d}$ given by

$$
g_{a b c d}=g_{a b} g_{c d}+g_{a c} g_{b d}+g_{a d} g_{b c}
$$

where $g_{a b}$ is an invariant metric on the Lie algebra. We prove in this section that if $g_{a b}$ is invertible, then the associated Chern-Simons theory is generic. This means, in particular, that the seven-dimensional theory with the choice (4.1) - or with any other choice of invariant tensor sufficiently close to it - is generic for any simple Lie algebra.

The constraint in this case reduces to the simpler form

$$
K_{a}=-F_{a \wedge} F_{\wedge}^{b} F_{b}=0
$$

where the internal indices are raised and lowered with $g_{a b}$. Here $F^{a}=\frac{1}{2} F_{i j}^{a} d x^{i} \wedge d x^{j}$, and we regard the constraint as a 6 -form. Similarly, $\Omega$ is a 4 -form given by

$$
\Omega_{a b}=-g_{a b} F_{\wedge}^{c} F_{c}-2 F_{a \wedge} F_{b} .
$$


We want to find solutions to Eq. (4.2) so that $\Omega$ has maximum rank. Thus, we are interested in the number of solutions of the null eigenvalue problem

$$
\Omega_{a b \wedge} V^{b}=0
$$

where $V^{b}$ is a 1 -form vector, and $F^{a}$ satisfies Eq. (4.2). We already know that this equation has six independent solutions which are of the form $V_{i}^{a}=F_{i j}^{a} \xi^{j}$. We will now show that there exists a solution to the constraint (4.2) for which $\Omega$ does not have any other null eigenvectors.

The constraint (4.2) is solved by the following expression for $F^{a}$ :

$$
F^{a}=f^{a} d x^{1} \wedge d x^{2}+g^{a} d x^{3} \wedge d x^{4}+h^{a} d x^{5} \wedge d x^{6},
$$

where

$$
f^{a}=x^{a}+\sqrt{3} y^{a}, \quad g^{a}=x^{a}-\sqrt{3} y^{a}, \quad h^{a}=x^{a}
$$

and $x^{a}, y^{a}$ are two vectors satisfying $x^{a} x_{a}=1=y^{a} y_{a}, x^{a} y_{a}=0$. Here we have assumed that $g_{a b}=\delta_{a b}$ only for simplicity. The analysis can be carried out for any invertible $g_{a b}$.

The matrix $\Omega$ evaluated for this solution is equal to

$$
\begin{aligned}
\Omega_{a b}= & 2\left(A_{a b} d x^{1} \wedge d x^{2} \wedge d x^{3} \wedge d x^{4}+B_{a b} d x^{1} \wedge d x^{2} \wedge d x^{5} \wedge d x^{6}\right. \\
& \left.+C_{a b} d x^{3} \wedge d x^{4} \wedge d x^{5} \wedge d x^{6}\right),
\end{aligned}
$$

where

$$
\begin{aligned}
& A_{a b}=\left(f^{c} g_{c}\right) g_{a b}+f_{a} g_{b}+f_{b} g_{a}, \\
& B_{a b}=\left(f^{c} h_{c}\right) g_{a b}+f_{a} h_{b}+f_{b} h_{a}, \\
& C_{a b}=\left(h^{c} g_{c}\right) g_{a b}+h_{a} g_{b}+h_{b} g_{a} .
\end{aligned}
$$

An immediate set of null eigenvectors of $\Omega$ comes from the observation that $h^{a}, g^{a}$ and $f^{a}$ are, respectively, null eigenvectors of $A, B$ and $C$. These eigenvectors are easily seen to correspond to the diffeomorphisms eigenvectors $\xi^{i} F_{i j}^{a}$.

To prove that this theory has maximum rank, it is now enough to prove that the matrices $A, B$ and $C$ do not have any further null eigenvectors. This is most easily shown by going to the particular basis in which

$$
x^{a}=(1,0,0, \ldots, 0), \quad y^{a}=(0,1,0, \ldots, 0) .
$$

Then, the vectors $f, g$ and $h$ have the form

$$
\begin{aligned}
& f^{a}=(1, \sqrt{3}, 0, \ldots, 0), \quad g^{a}=(1,-\sqrt{3}, 0, \ldots, 0), \\
& h^{a}=(1,0,0, \ldots, 0)
\end{aligned}
$$

and $A, B$ and $C$ have the block form 


$$
\begin{aligned}
& A=\left(\begin{array}{cc|c}
0 & 0 & 0 \\
0 & -8 & 0 \\
\hline 0 & 0 & -2 I
\end{array}\right), \quad B=\left(\begin{array}{cc|c}
3 & \sqrt{3} & 0 \\
\sqrt{3} & 1 & 0 \\
\hline 0 & 0 & I
\end{array}\right) \\
& C=\left(\begin{array}{cc|c}
3 & -\sqrt{3} & 0 \\
-\sqrt{3} & 1 & 0 \\
\hline 0 & 0 & I
\end{array}\right),
\end{aligned}
$$

where $I$ is the identity in the $(N-2) \times(N-2)$ subspace orthogonal to $x^{a}$ and $y^{a}$. Accordingly, $A, B$ and $C$ have rank $N-1$ showing that each of them has only one null eigenvector. These eigenvectors are, respectively, $h^{a}, g^{a}$ and $f^{a}$.

Thus, we have proved that the seven-dimensional Chern-Simons theory defined by the invariant tensor given in Eq. (4.1) provides another example of generic theories.

\section{The $(N=1)$ Abelian theory}

The $N=1$ theory was first studied in Ref. [18] where the absence of degrees of freedom for this theory was pointed out. Here we shall analyze this theory along the lines introduced in Section 2. Our main goal is to display the differences between the $N=1$ and $N>1$ theories.

As it was pointed out at the end of Section 2.4 , the $N=1$ theory needs a special treatment. We defined in that section the generic solutions of the constraint as those for which the matrix $F_{i j}$ has the maximum possible rank $2 n-2$.

Let us split the $2 n$ spatial coordinates $x^{i}$ into $\left(x^{\alpha}, x^{p}\right)$ where $x^{\alpha}=\left(x^{1}, x^{2}\right)$ and $x^{p}=\left(x^{3}, x^{4}, \ldots, x^{2 n}\right)$, so that the gauge field takes the form

$$
A_{i} d x^{i}=A_{\alpha} d x^{\alpha}+A_{p} d x^{p}
$$

and the curvature can be written as

$$
F_{i j} d x^{i} \wedge d x^{j}=F_{\alpha \beta} d x^{\alpha} \wedge d x^{\beta}+2 F_{\alpha p} d x^{\alpha} \wedge d x^{p}+F_{p q} d x^{p} \wedge d x^{q} .
$$

The generic condition for the $N=1$ theory can be implemented by requiring that the $(2 n-2) \times(2 n-2)$ matrix $F_{p q}$ appearing in Eq. (5.2) is invertible, i.e.

$$
\operatorname{det} F_{p q} \neq 0 \text {, }
$$

so that $F_{i j}$ has the maximum rank $2 n-2$. By a change of coordinates, one can always make a generic $F_{i j}$ to fulfill the condition (5.3).

\subsection{Dirac brackets and first class algebra}

Once the maximum rank condition over $F_{p q}$ is imposed, the constraints $\phi^{i}=\left(\phi^{\alpha}, \phi^{p}\right)$ split naturally into first and second class. To see this, we first note that $\Omega^{\alpha \beta}$ (the projection of $\Omega^{i j}$ along the coordinates $x^{\alpha}$ ) can be written as 


$$
\Omega^{\alpha \beta}=-\frac{1}{2^{n-1}} \epsilon^{\alpha \beta} f
$$

where $f$ is the Pfaffian of $F_{p q}$,

$$
f \equiv \epsilon^{p_{1} \cdots p_{2 n-2}} F_{p_{1} p_{2}} \cdots F_{p_{2 n-3} p_{2 n-2}}=\sqrt{\operatorname{det} F_{p q}}
$$

and $\epsilon^{\alpha \beta}$ is the Levi-Civita tensor in the two-dimensional manifold labeled by the coordinates $x^{\alpha}$. Since the determinant of $F_{p q}$ is different from zero, the $2 \times 2$ matrix $\Omega^{\alpha \beta}$ is invertible. Let $J$ be its inverse:

$$
J_{\alpha \beta}=\frac{2^{n-1}}{f} \epsilon_{\alpha \beta},
$$

which satisfies $J_{\alpha \beta} \Omega^{\beta \gamma}=\delta_{\alpha}^{\gamma}$.

The invertibility of $\Omega^{\alpha \beta}$ implies, from Eq. (2.14), that the two constraints $\phi^{\alpha}$ are second class. Consequently, their associated Lagrange multipliers $u_{\alpha}$ can be solved from Eq. (2.18),

$$
u_{\alpha}=J_{\alpha \beta} \Omega^{\beta p} u_{p}
$$

As we have seen, one may take as first class constraints the $2 n$ combinations $H_{i}=$ $F_{i j} \phi^{j}$. In the $N=1$ case, there are only $2 n-2$ independent constraints among the $H_{i}$ 's, since the matrix $F_{i j}$ is of rank $2 n-2$. If we recall that the matrix $F_{p q}$ is invertible, we can take the independent first class constraints to be $H_{p}=F_{p q} \phi^{q}+F_{p \alpha} \phi^{\alpha}$. The system of constraints $\left(\phi^{\alpha}, H_{p}\right)$ provides a system equivalent to the system $\left(\phi^{j}\right)$, in which the constraints are manifestly split into second and first class. Upon elimination of the second class constraints, we obtain the corresponding Dirac bracket

$$
\{A, B\}^{*}=\{A, B\}-\int_{\Sigma} d z\left\{A, \phi^{\alpha}(z)\right\} J_{\alpha \beta}(z)\left\{\phi^{\beta}(z), B\right\} .
$$

The smeared generators $G(\lambda)=\int_{\Sigma} \lambda G$ and $\bar{H}(\xi)=\int_{\Sigma} \xi^{p} \bar{H}_{p}$, where $\bar{H}_{p}=F_{p q} \phi^{q}+$ $A_{p} G$ satisfy the Dirac bracket algebra,

$$
\begin{aligned}
& \{G(\lambda), G(\eta)\}^{*}=0, \\
& \{\bar{H}(\xi), G(\lambda)\}^{*}=G\left(\xi^{p} \partial_{p} \lambda\right), \\
& \{\bar{H}(\xi), \bar{H}(\zeta)\}^{*}=\bar{H}([\xi, \zeta]),
\end{aligned}
$$

where $[\xi, \zeta]^{p}=\xi^{q} \zeta^{p}, q-\zeta^{q} \xi^{p}, q$ is the Lie bracket of the two vectors $\xi^{p}$ and $\zeta^{q}$. The above algebra is self-explanatory; $G$ generates gauge transformations and $\bar{H}_{p}$ generates diffeomorphisms in the $x^{p}$ directions. Eq. (5.10), on the other hand, tells us that $G$ transforms as a scalar under diffeomorphisms.

This completes the problem of relating the first class algebra with the symmetries of the Chern-Simons action. A different question is whether the above constraints encode all the symmetries of the action. Two evident missing pieces are diffeomorphisms along the $x^{\alpha}$ directions and time-like diffeomorphisms. It turns out that these symmetries are 
generated by the above constraints. The proof of this statement is straightforward. The key point is the fact that the equations of motion trivialize the time-like (as in the case $N>1$ ) and $x^{\alpha}$-diffeomorphisms. That is, when acting on the space of solutions of the equations of motion, these symmetries reduce to the identity. For this reason, there are no independent first class constraints associated to them. In a canonical language, the generators of time-like and $x^{\alpha}$-diffeomorphisms are linear combinations of the generators of gauge transformations and $x^{p}$-diffeomorphisms. This is reminiscent of the $2+1$ theory where the whole diffeomorphism invariance can be expressed in terms of the local gauge transformations.

There is thus a gradation that can be summarized as follows: in three dimensions, the diffeomorphisms can be expressed in terms of the internal gauge transformations for any choice of the gauge group. In higher dimensions, some of the diffeomorphisms become independent gauge symmetries (in the generic case). These are $2 n-2$ of the spatial diffeomorphisms if the gauge group is one-dimensional, and all the spatial diffeomorphisms otherwise. The time-like diffeomorphisms are not independent gauge symmetries in any (generic) case; they can always be expressed in terms of the other symmetries.

\subsection{Absence of local degrees of freedom}

Having determined the first and second class constraints we can now proceed to count the number of local degrees of freedom of this theory. We have $2 n$ canonical variables $A_{i}$ and $2 n$ canonical momenta $p^{i}$. To this number, $4 n$, we subtract the number of second class constraints, namely 2 , and twice the number of first class constraints, $2 \times(2 n-1)$, so that

$$
2 \mathcal{N}=4 n-2-2 \times(2 n-1)=0 .
$$

Thus, there are no local degrees of freedom. This is a pure topological field theory and the only degrees of freedom that may be present are the global ones. We must stress again, however, that this is a peculiarity of the $N=1$ theory, which is in that sense a poor representative of the general case.

\subsection{Reduced action}

It is instructive to write down the reduced action once the second class constraints have been solved. Since the constraints $\phi^{\alpha}$ are linear in the momenta, this is easily achieved. Upon inserting the solution inside the action, one obtains a reduced action for the relevant dynamical fields and Lagrange multipliers,

$$
S\left[A_{\alpha}, A_{p}, p^{p} ; A_{0}, N^{p}\right]=\int_{\mathbb{R}} \int_{\Sigma}\left[l^{\alpha}\left(A_{\alpha}, A_{p}\right) \dot{A}_{\alpha}+p^{p} \dot{A}_{p}-A_{0} G-N^{p} \bar{H}_{p}\right],
$$

which must be varied with respect to all its arguments. 
The symplectic structure is not canonical due to the presence of the factor $l^{\alpha}\left(A_{\alpha}, A_{p}\right)$ in the first kinetic term. The symplectic form

$$
\omega=\frac{1}{2} \int_{\Sigma}\left(\Omega^{\alpha \beta} \delta A_{\alpha^{\wedge}} \delta A_{\beta}+2 \Omega^{\alpha p} \delta A_{\alpha^{\wedge}} \delta A_{p}+2 \delta A_{p^{\wedge}} \delta p^{p}\right)
$$

is, by construction, invertible and its inverse provides the Dirac bracket

$$
\begin{array}{lll}
\left\{A_{\alpha}, A_{\beta}\right\}^{*}=J_{\alpha \beta}, & \left\{A_{\alpha}, A_{p}\right\}^{*}=0, & \left\{A_{\alpha}, p^{p}\right\}^{*}=J_{\alpha \beta} \Omega^{\beta p}, \\
\left\{A_{p}, A_{q}\right\}^{*}=0, & \left\{A_{p}, p^{q}\right\}^{*}=\delta_{p}^{q}, & \left\{p^{p}, p^{q}\right\}^{*}=0,
\end{array}
$$

which is equivalent to that defined in Eq. (5.8), in agreement with the general theory [2].

\subsection{Comparison with superparticle}

The canonical analysis of the $U(1)$-Chern-Simons theory in higher dimensions presents many similarities with that of the superparticle (see, e.g., Ref. [9] and references therein). In both cases, although the original action is manifestly covariant to begin with, one cannot reach a complete canonical formulation without breaking explicitly this manifest covariance. This is because one cannot isolate covariantly the second class constraints [8]. Furthermore, although one can write down a complete set of first class constraints that transform covariantly (here, the constraints $F_{i j} \phi^{j}$ ), these first class constraints are redundant, implying in a BRST treatment the presence of ghosts of ghosts. Again, one cannot isolate covariantly a complete, irreducible, set of first class constraints.

There is another interesting similarity: if one chooses to work with the covariant, redundant, first class constraints $F_{i j} \phi^{j}$, one finds that the reducibility identities are

$$
\mu^{k i} F_{i j} \phi^{j}=0
$$

(on the constraint surface $G=0$ ), with

$$
\mu^{k i}=\epsilon^{k i i_{1} \cdots i_{2 n-2}} F_{i_{1} i_{2}} \ldots F_{i_{2 n-3} i_{2 n-2}} .
$$

These reducibility identities, in turn, are not independent since $F_{l k} \mu^{k i}=0$ (weakly), and this reducibility of the reducibility is itself not irreducible, etc. One is thus led to an infinite tower of reducibility identities, requiring an infinite set of ghosts of ghosts in the BRST formulation, exactly as in the superparticle case $[9,10]$.

\section{The $G \times U(1)$ theory}

The Hamiltonian analysis performed so far in the $N>1$ case is incomplete because (i) the second class constraints have not yet been eliminated and (ii) in the case of 
manifolds with boundaries, it is known that the Hamiltonian has to be supplemented with some boundary terms [19], a problem not yet discussed. These two issues are cumbersome on the computational side - even though conceptually easy - if one works with an arbitrary Lie group $G$ and, actually, they cannot be treated in a general covariant way. It is surprising, therefore, that a drastic simplification takes place if one couples a $U$ (1) factor to the group $G$. To avoid unessential technical difficulties, we shall restrict our attention to that case, which illustrates all the conceptual features. In this section, we solve all second class constraints and compute the Dirac bracket. In the next section, we deal with the boundary terms necessary to make the Hamiltonian well-defined. For simplicity, we work explicitly in five dimensions but we shall indicate how the results obtained here can be extended to any odd-dimensional space-time.

\subsection{The invariant tensor for $G \times U(1)$}

Consider the Chern-Simons action in five dimensions for the Lie group $G \times U(1)$. (In this section capital Latin indices $A, B, \ldots$ run over $G \times U(1)$. Small Latin indices $a, b, \ldots$ run over $G$, and 1 denotes $U(1)$.) It is straightforward to see that the invariance condition on the tensor $g_{A B C}$ implies the following restrictions on its components. The components $g_{a b c}, g_{a b 1}$ and $g_{a 11}$ must separately be invariant under the adjoint action of $G$, and $g_{111}$ is an arbitrary constant.

We shall now impose three extra conditions on the group $G \times U(1)$ and its invariant tensors. First we assume that $g_{a 11}$ is zero. Usually, this is not an additional requirement, because, in general, there is no vector invariant vector under the adjoint action of $G$. Second, we assume that $G$ admits an invariant non-degenerate quadratic form $g_{a b}$, as it is the case if $G$ is semisimple, and we take

$$
g_{a b 1}=g_{a b} .
$$

Finally, we impose

$$
g_{111}=0 \text {. }
$$

This condition is justified on simplicity grounds, since, as we shall see below, it allows for a simple separation between first and second class constraints. Note that the gauge fields associated with $G$ and $U(1)$ respectively are not decoupled in the action, because $g_{a b 1} \neq 0$.

\subsection{Dirac brackets}

An immediate consequence of this choice for the invariant tensor is that the second class constraints can be explicitly isolated and solved, at least in a generic region of phase space.

The constraint equations $\left(K_{A}=-\frac{1}{2} g_{A B C} F^{B} \wedge F^{C}=0\right)$, in this case, are

$$
K_{a}=-\frac{1}{2} g_{a b c} F^{b} \wedge F^{c}-F^{1} \wedge F_{a}=0 \quad(A=a),
$$




$$
K_{1}=-\frac{1}{2} g_{a b} F_{\wedge}^{a} F^{b}=0 \quad(A=1) .
$$

An obvious solution for these equations is $F^{a}=0$ and $F^{1}$ completely arbitrary. The matrix $\Omega$ evaluated on this particular solution has the block form

$$
\left.\Omega_{A B}^{i j}\right|_{F^{a}=0}=\left(\begin{array}{c|c}
0 & 0 \\
\hline 0 & -\frac{1}{2} g_{a b} \epsilon^{i j k l} F_{k l}^{1}
\end{array}\right) .
$$

If the matrix $F_{i j}^{1}$ is non-degenerate, then $\Omega$ (evaluated on that particular solution) has the maximum rank $4 N-4$. Hence, we have proved that the $G \times U(1)$ theory is generic in the sense described in Section 2.4. Of course, a degenerate $F^{1}$ provides also a solution for the equations of motion. Such a solution, however, belongs to a different branch of the theory with a smaller number of local degrees of freedom.

Since the solution $F^{a}=0$ and $F^{1}$ non-degenerate is such that the matrix $\Omega$ has maximum rank, a sufficiently small perturbation around it will not change this rank (the rank is a semi-continuous function from below). Thus, on the portion of phase space around the solution $\left\{F^{a}=0, \operatorname{det} F^{1} \neq 0\right\}$, the sub-matrix $\Omega_{a b}^{i j}$ is invertible. This means that among the primary constraints $\phi_{A}^{i}$, the subset $\phi_{a}^{i}$ is second class. Moreover, since the matrix $F_{i j}^{1}$ is invertible in an open region around the above solution, one can replace the set of constraints $\left(\phi_{1}^{i}, \phi_{a}^{i}\right)$ by the equivalent set $\left(H_{i}, \phi_{a}^{i}\right)$, where $H_{i}=F_{i j}^{1} \phi_{1}^{j}+F_{i j}^{a} \phi_{a}^{j}$ are the first class constraints that generate the diffeomorphisms. In this new set, the constraints are separated into first class and second class. The technical simplification that motivated the choice of group $G \times U(1)$ appears precisely here: the separation of the constraints into first and second classes can be easily achieved.

Once the second class constraints have been isolated one can compute the Dirac bracket. We define the inverse of the matrix $\Omega_{a b}^{i j}$ by

$$
\Omega_{a b}^{i j} J_{j k}^{a c}=\delta_{k}^{i} \delta_{b}^{c}
$$

The Dirac bracket among two phase space functions $A$ and $B$ is then given by

$$
\{A, B\}^{*}=\{A, B\}-\int_{\Sigma} d z\left\{A, \phi_{a}^{i}(z)\right\} J_{i j}^{a b}(z)\left\{\phi_{b}^{j}(z), B\right\},
$$

which gives the following Dirac bracket relations among the elementary variables:

$$
\begin{array}{lll}
\left\{A_{i}^{a}, A_{j}^{b}\right\}^{*}=J_{i j}^{a b}, & \left\{A_{i}^{a}, A_{j}^{1}\right\}^{*}=0, & \left\{A_{i}^{a}, p_{1}^{j}\right\}^{*}=J_{i k}^{a b} \Omega_{b 1}^{k j}, \\
\left\{A_{i}^{1}, p_{1}^{j}\right\}^{*}=\delta_{i}^{j}, & \left\{A_{i}^{1}, A_{j}^{1}\right\}^{*}=0, & \left\{p_{1}^{i}, p_{1}^{j}\right\}^{*}=0 .
\end{array}
$$

The brackets of the variable $A_{i}^{1}$ with the other variables are simple. However, the brackets of the variables $A_{i}^{a}$ among themselves and with the $p_{1}^{j}$,s are more involved. In the quantum theory, the $A_{i}^{a}$ 's are not commuting operators. An interesting question not investigated here is to find an explicit realization of the Dirac bracket algebra in terms of commutators. This question is not straightforward, because the left-hand sides of the Dirac brackets among the basic variables are not $c$-numbers. 
If we now work with the Dirac bracket, we can set the second class constraints strongly equal to zero and keep only the first class ones in the formalism. These are given by $G_{a}, G_{1}$ and $H_{i}$ defined in Eqs. (2.11) and (2.20), respectively and are the generators of internal gauge transformations and of spatial diffeomorphisms. After the second class constraints have been set equal to zero, these generators simplify to

$$
\begin{aligned}
& G_{a}=-K_{a}, \\
& G_{1}=-K_{1}+\partial_{i} \phi_{1}^{i}, \\
& H_{i}=F_{i j}^{1} \phi_{1}^{i} .
\end{aligned}
$$

One can easily check that, in the Dirac bracket, $G_{a}$ and $G_{1}$ given above satisfy the Lie algebra of $G \times U(1)$ and $H_{i}$ satisfy the algebra of diffeomorphisms up to a gauge transformation.

The same analysis can be performed in all odd dimensions greater than five provided that the invariant tensor $g_{A_{1} A_{2} \ldots A_{n+1}}$ satisfies the conditions $g_{11 \ldots 1}=0, g_{a 1 \ldots 1}=0, g_{a b 11 \ldots 1}=$ $g_{a b}$ (with $g_{a b}$ an invertible invariant metric), while all the other components are invariant tensors of $G$. Again, the configuration $F^{a}=0$, det $F^{1} \neq 0$ is a maximum rank solution for the constraint equations and therefore the theory is generic.

\section{Global symmetries and $\mathrm{WZW}_{4}$ algebras}

We now turn to the problem of boundary conditions and boundary terms when the spatial manifold has a boundary, again in the simple context of a gauge group of the form $G \times U(1)$. There is a special motivation for doing this. Indeed, a four-dimensional analog of the WZW Lagrangian exists [6]. This model is characterized by a symmetry algebra that generalizes the familiar Kac-Moody algebra in two dimensions, and which has been called $\mathrm{WZW}_{4}$ algebra [6,7]. It is a natural question to see whether this algebra can be obtained from a Chern-Simons theory in five dimensions just as the Kac-Moody algebra is generated from the $2+1$ Chern-Simons theory [4] (see also Ref. [20]). This problem has already been studied in the literature in interesting works that considered modifications of the Chern-Simons action. In Ref. [5], the Yang-Mills action was added to the Chern-Simons action in order to make the symplectic structure simpler. This procedure, however, breaks the diffeomorphism invariance. In Ref. [6], a Chern-Simons theory coupled to a fixed Kälher form is considered. This procedure also breaks part of the diffeomorphism invariance of the theory. Finally, 4D currents arising from the Abelian $N=1$ Chern-Simons theory have been studied in Ref. [22].

It is the purpose of this section to prove that the issue of global charges in pure Chern-Simons theories can be analyzed in the full unmodified non-Abelian theory without making any particular assumptions (other than the boundary conditions). In this paper, however, we do not study the effective Lagrangians arising at the boundary, but only the algebras. 
As usual in any gauge theory, in the presence of boundaries, the gauge symmetries split into 'proper' and 'improper' gauge symmetries. The proper symmetries are those transformations which are generated by the constraints through Poisson brackets. Their generators are thus weakly zero. Improper symmetries, on the other hand, are generated by the constraints supplemented with a (non-vanishing) boundary term. They should be viewed as global symmetries. After the gauge is fixed and the constraints are strongly set equal to zero, these boundary terms, the charges, 'survive' and satisfy a well-defined Dirac bracket algebra at the boundary.

Consider the Chern-Simons theory for the group $G \times U(1)$ discussed in the last section and consider a gauge transformation along $G$ with a parameter $\eta^{a}$. We define

$$
G_{Q}(\eta)=\int_{\Sigma} \eta^{a} G_{a}+Q(\eta)
$$

where the charge $Q$ has to be adjusted so that $G_{Q}(\eta)$ generates the transformation $\delta A^{a}=-D \eta^{a}$ for the gauge field, even at the boundary. By direct application of the Dirac bracket defined in Eq. (6.7), the transformation induced by $G_{Q}(\eta)$ on $A_{i}^{a}$ is given by

$$
\delta_{\eta} A_{i}^{a}(x)=\left\{A_{i}^{a}(x), G_{Q}(\eta)\right\}^{*}=J_{i j}^{a b}(x) \frac{\delta G_{Q}(\eta)}{\delta A_{j}^{b}(x)} .
$$

Recall that since we are working with the Dirac brackets, the generator $G_{a}$ (for the group $G \times U(1))$ has the simple expression $G_{a}=-K_{a}$.

We need to compute the functional derivative of $G_{Q}(\eta)$. From the definition of $G_{a}$, one finds that if the charge $Q$ satisfies the equation

$$
\delta Q=\int_{\partial \Sigma} \eta^{a}\left(g_{a b c} F^{a} \wedge \delta A^{b}+g_{a b} F^{1} \wedge \delta A^{b}\right),
$$

then, the derivative of $G_{Q}(\eta)$ is well-defined and given by

$$
\frac{\delta G_{Q}(\eta)}{\delta A_{i}^{a}(x)}=-\Omega_{a b}^{i j}(x) D_{j} \eta^{b}(x) .
$$

Formula (7.2), together with Eq. (6.6) gives $\delta A_{i}^{a}=-D_{i} \eta^{a}$, as expected.

There are two remaining things to be checked before we can fully promote $G_{Q}(\eta)$ to the generator of gauge transformation with a parameter $\eta$. First, we need to integrate relation (7.3) in order to extract from it the value of $Q$. Second, we need to compute the algebra of $G_{Q}(\eta)$.

In order to integrate Eq. (7.3), we shall impose the following boundary conditions:

$$
F^{a}=0, \quad F^{1}=\omega \equiv \text { fixed } 2 \text {-form (at the boundary). }
$$

The charge $Q$ for these boundary conditions is then given by

$$
Q(\eta)=\int_{\partial \Sigma} \omega \wedge A^{a} \eta^{b} g_{a b}
$$


Now we turn to the problem of the algebra of $G_{Q}(\eta)$. The Dirac bracket of two generators $G_{Q}(\eta)$ and $G_{Q}(\rho)$ is

$$
\left\{G_{Q}(\eta), G_{Q}(\rho)\right\}^{*}=\int_{\Sigma} \Omega_{a b^{\wedge}} D \eta^{a} \wedge D \rho^{b}
$$

After an integration by parts, keeping all boundary terms, and using the Jacobi identity for the structure constants $f_{b c}^{a}$ of the group $G$, the right-hand side of Eq. (7.7) can be written as

$$
\left\{G_{Q}(\eta), G_{Q}(\rho)\right\}^{*}=\int_{\Sigma}[\eta, \rho]^{a} G_{a}+\int_{\partial \Sigma} \omega \wedge[\eta, \rho]_{a} A^{a}+\int_{\partial \Sigma} \omega \wedge \eta_{a} d \rho^{a}
$$

where $[\eta, \rho]^{a}=f_{b c}^{a} \eta^{b} \rho^{c}$. The boundary term on the right-hand side has two pieces. The first term is precisely the charge $Q([\eta, \rho])$ that regularizes the bulk integral. The second term, on the other hand, does not depend on the fields that are varied at the boundary and, therefore, is a central term. The algebra (7.8) can then be rewritten in its final form,

$$
\left\{G_{Q}(\eta), G_{Q}(\rho)\right\}^{*}=G_{Q}([\eta, \rho])+\int_{\partial \Sigma} \omega \wedge \eta_{a} d \rho^{a}
$$

As we can see, this algebra is not homomorphic to the original algebra of $G$ but it is a non-trivial central extension of it. (The possibility of non-trivial central charges in the canonical realization of global charges given by surface integrals was demonstrated in general in Ref. [21].) This algebra was first obtained in Ref. [6]. In that paper, the role of $F^{1} \equiv \omega$ was played by a non-dynamical Kähler form while here, it appears as the curvature of the $U(1)$ factor.

The algebra (7.9) is a natural generalization of the Kac-Moody algebra existing in two dimensions. We have shown in this section that, as one could have expected, ChernSimons theory in five dimensions generates a 'conformal' theory on the four-dimensional boundary.

This analysis can be repeated in higher dimensions with an invariant tensor that fulfills the conditions spelled out in the previous section. One finds that the algebra of the charges is simply

$$
\left\{G_{Q}(\eta), G_{Q}(\rho)\right\}^{*}=G_{Q}([\eta, \rho])+\int_{\partial \Sigma} \omega \wedge \omega \wedge \cdots \wedge \omega \wedge \eta_{a} d \rho^{a}
$$

where $\omega$ is a fixed two-form. In analogy with the four-dimensional terminology, it may be called ' $\mathrm{WZW}_{2 n}$ algebra'. 


\section{Conclusions}

We have shown that higher dimensional Chern-Simons theories, even though constructed along the same topological pattern as in $2+1$ dimensions, have local degrees of freedom provided that the invariant tensor that enters the action fulfills an appropriate generic condition. This condition implies that there are no accidental gauge symmetries, so that the number of gauge symmetries grows more slowly with the dimension of the gauge group than with the number of dynamical variables. This result cannot be anticipated by analyzing the case of a single Abelian field, which is not representative of the general case.

Chern-Simons theories in higher dimensions provide, accordingly, examples of theories that are generally covariant without involving a dynamical metric, and yet, that carry local dynamical degrees of freedom. Therefore, they constitute counter-examples to the belief that such theories can contain only global or surface degrees of freedom.

We have illustrated the presence of local degrees of freedom with examples in seven dimensions. These examples complement the five-dimensional examples given in Ref. [1]. We have also applied the analysis to Lovelock-Chern-Simons gravity in any odd dimensions and have established by a mere count of the number of local degrees of freedom that the first order (Palatini) and the second order (metric) formalisms are not equivalent.

We have also shown that the time-like diffeomorphisms do not lead to independent constraints. The implications of this remarkable feature for the quantum theory remain to be explored. As a first step, it would be interesting to investigate how the loop representation must be defined when the connection obeys the non-trivial Dirac brackets computed above.

We have finally studied the global charges that naturally arise in the presence of boundaries and have shown that, at least for the gauge group $G \times U(1)$, the gauge generators satisfy the $\mathrm{WZW}_{4}$ algebra in five dimensions, just as the Kac-Moody algebra arises in manifolds with boundaries for Chern-Simons theories in $2+1$ dimensions. This $\mathrm{WZW}_{4}$ algebra can be generalized to the $\mathrm{WZW}_{2 n}$ algebras, which appear again as global symmetry algebras on the boundary for Chern-Simons theories in dimension $2 n+1$.

\section{Acknowledgements}

M.H. is grateful to LPTHE (Universités Paris VI and Paris VII) for kind hospitality. M.B. is partially supported by a grant from Fundación Andes (Chile), grants 1930910-93 and 1960065-94 from FONDECYT (Chile), and by institutional support to the Centro de Estudios Científicos de Santiago provided by SAREC (Sweden) and a group of Chilean private companies (EMPRESAS CMPC, CGE, COPEC, MINERA LA ESCONDIDA, NOVAGAS Transportandores de Chile, ENERSIS, BUSINESS DESIGN ASS., XEROX Chile). L.J.G. was supported by funds provided by DGICYT and MEC (Spain) under 
Contract Adjunct to the Project No. PB94-0107. The work of M.H. is partially supported by research funds from F.N.R.S. (Belgium) and a research contract with the Commission of the European Community.

\section{References}

[1] M. Bañados, L.J. Garay and M. Henneaux, Phys. Rev. D 53 (1996) R593.

[2] M. Henneaux and C. Teitelboim, Quantization of Gauge Systems (Princeton University Press, Princeton, 1992).

[3] A. Ashtekar, Phys. Rev. Lett. 57 (1986) 2244; Phys. Rev. D 36 ( 1987) 1587;

C. Rovelli and L. Smolin, Phys. Rev. Lett. 61 (1988) 1155; Nucl. Phys. B 331 (1990) 80;

Brügmann, R. Gambini and J. Pullin, Phys. Rev. Lett. 68 (1992) 431;

R. Gambini and J. Pullin, [gr-qc/9603019].

141 G. Moore and N. Seiberg, Phys. Lett. B 220 (1989) 422;

S. Elizur, G. Moore, A. Schwimmer and N. Seiberg, Nucl. Phys. B 326 (1989) 108;

E. Witten, Commun. Math. Phys, 121 (1989) 351.

[5] G.V. Dunne and C.A. Trugenberger, Phys. Lett. B 248 (1990) 311;

A.P. Balachandran, G. Bimonti, K.S. Gupta and A. Stern, Int. J. Mod. Phys. A 7 (1992) 4655.

[6] V.P. Nair and J. Schiff, Phys. Lett. B 246 (1990) 423; Nucl. Phys. B 371 (1992) 329.

[7] A. Losev, G. Moore, N. Nekrasov and S. Shatashvili, Four-dimensional avatars of two-dimensional RCFT, [hep-th/9509151].

[8] I. Bengtsson and M. Cederwall, Goteborg preprint GOTEBORG-84-21 (1984).

19] L. Brink and M. Henneaux, Principles of String Theory (Plenum Press, New York, 1988);

L. Brink, M. Henneaux and C. Teitelboim, Nucl. Phys. B 293 (1987) 505.

[10] R.E. Kallosh, Phys. Lett. B 195 (1987) 369.

[11] R. Jackiw, Phys. Rev. Lett 41 (1978) 1635.

[12] D. Lovelock, J. Math. Phys. 12 (1971) 498.

[13] A. Achúcarro and P. Townsend, Phys. Lett. B 180 (1986) 89.

[14] E. Witten, Nucl. Phys. B 311 (1988) 46.

[15] A.H. Chamseddine, Nucl. Phys. B 346 (1990) 213; Phys. Lett. B 233 (1989) 291.

[16] M. Bañados, C. Teitelboim and J. Zanelli, Phys. Rev. D 49 (1994) 975;

see also J.J. Giambiagi Festschriff, H. Falomir, R. Gamboa, P. Leal and F. Shaposnik, eds. (World Scientific, Singapore, 1991).

[17] C. Teitelboim and J. Zanelli, Class. Quant. Grav. 4 (1987) L125; in: Constraint Theory and Relativistic Dynamics, G. Longhi and L. Lussana, eds. (World Scientific, Singapore, 1987).

[18] R. Floreanini, R. Percacci and R. Rajaraman, Phys. Lett. B 231 (1989) 119;

R. Floreanini and R. Percacci, Phys. Lett. B 224 (1989) 291.

[19] T. Regge and C. Teitelboim, Ann. Phys. (NY) 88 (1974) 286.

[20] M. Bañados, Phys. Rev. D 52 (1995) 5816;

O. Coussaert, M. Henneaux and P. van Driel, Class. Quant. Grav. 12 (1995) 2961.

[21] J.D. Brown and M. Henneaux, J. Math. Phys. 27 (1986) 489; Commun. Math. Phys. 104 (1986) 207.

[22] K.S. Gupta and A. Stern, Nucl. Phys. B 442 (1995) 157. 